\title{
Óleos encapsulados: perfil de ácidos graxos e contaminação por hidrocarbonetos policíclicos aromáticos
}

Encapsulated oils: fatty acid profile and contamination by polycyclic aromatic hydrocarbons

\author{
M. M. M. Kus-Yamashita ${ }^{1 *}$; S. A. Silva²; A. P. Almeida ${ }^{2}$; S. A. Pimentel ${ }^{1}$ \\ ${ }^{l}$ Núcleo de Química, Física e Sensorial/Centro de Alimentos, Instituto Adolfo Lutz, 01246-000, São Paulo-SP, Brasil \\ ${ }^{2}$ Núcleo de Contaminantes Orgânicos/Centro de Contaminantes, Instituto Adolfo Lutz, 01246-000, São Paulo-SP, \\ Brasil \\ *mahyara.kus@ial.sp.gov.br \\ (Trabalho avaliado e selecionado pela Comissão do III CTOCTA)
}

\begin{abstract}
Os óleos vegetais encapsulados podem ser extraídos a frio e ter como origem fontes não convencionais, reconhecidos por preservarem compostos bioativos característicos e, alguns deles, serem fontes de ácidos graxos essenciais. No entanto, são produtos geralmente onerosos e por este motivo, passíveis de adulteração com óleos de menor qualidade. O objetivo deste trabalho foi avaliar a identidade e a contaminação por hidrocarbonetos policíclicos aromáticos (HPAs) de óleos encapsulados. Foram avaliadas catorze amostras, de diferentes tipos e marcas, comercializadas na cidade de São Paulo. Os ensaios realizados incluíram perfil de ácidos graxos e HPAs [benzo(a)antraceno, criseno, benzo(b)fluoranteno e benzo(a)pireno]. Duas amostras (14\%) foram consideradas insatisfatórias quanto aos perfis de ácidos graxos, incluindo óleos de coco e borragem. Quanto aos HPAs, as concentrações para o benzo(a)pireno variaram de $<\mathrm{LQ}$ a $0,51 \mu \mathrm{g} / \mathrm{kg}$ e, para a soma dos $4 \mathrm{HPAs}$, de $<\mathrm{LQ}$ a $5,83 \mu \mathrm{g} / \mathrm{kg}$. A avaliação e monitoramento destes óleos encapsulados deve ser constante, considerando o frequente consumo pela população e a possibilidade dos mesmos estarem adulterados.
\end{abstract}

Palavras-chave: óleos não convencionais, identidade, contaminação.

Encapsulated vegetable oils can be cold extracted and come from non-conventional sources, recognized for preserving characteristic bioactive compounds and, some of them, being sources of essential fatty acids. However, they are generally expensive products and for this reason, can be adulterated with lower quality oils. The objective of this work was to evaluate the identity and contamination by polycyclic aromatic hydrocarbons (PAHs) of encapsulated oils. Fourteen samples of different types and brands, commercialized in the city of São Paulo were evaluated. The analyses included the fatty acids profile and PAHs [benzo(a)anthracene, chrysene, benzo(b)fluoranthene, and benzo(a)pyrene]. Two samples (14\%) were considered unsatisfactory in terms of fatty acid profiles, including coconut, and borage oils. As for PAHs, the concentrations for benzo(a)pyrene ranged from $<\mathrm{LQ}$ to $0.51 \mu \mathrm{g} / \mathrm{kg}$ and, for the $4 \mathrm{PAHs}$ sum, from $<\mathrm{LQ}$ to $5.83 \mu \mathrm{g} / \mathrm{kg}$. The evaluation and monitoring of these encapsulated oils must be constant, considering the frequent consumption by the population and the possibility of them being adulterated.

Key-words: unconventional oils, identity, contamination.

\section{INTRODUÇÃO}

Diversos óleos têm sido usados na alimentação com papel considerável à promoção da saúde, devido à presença de componentes funcionais como compostos fenólicos e ácidos graxos (AG). Em especial, os ácidos graxos poli-insaturados ômega-3 e ômega-6 vêm ganhando importância como alimento, e devem estar presentes na dieta em grandes quantidades, uma vez que o corpo humano não consegue sintetizá-los. Ácidos graxos como o linolênico (ALA), eicosapentaenóicos (EPA) e docosahexaenóicos (DHA) têm potenciais benefícios à saúde, incluindo propriedades anti-inflamatórias, antimicrobianas, antitrombóticas, anti-hipertensivas, antienvelhecimento, anticâncer e antidiabéticas [1].

Considerando os benefícios, estes óleos têm uma demanda considerável e possuem valor elevado, uma vez que alguns óleos vegetais são obtidos por prensagem a frio e possuem um rendimento no processo inferior quando comparados aos demais óleos. Desta forma, podem ser propícios à adulteração, e misturados com outros óleos vegetais mais baratos, com a intenção de 
obter lucro potencial. No entanto, esta adulteração pode causar diversos problemas que afetam a saúde do consumidor [2].

São vários os ensaios usados para caracterizar a identidade de óleos vegetais, incluindo ácidos graxos, esteróis, tocoferóis, entre outros. Em alguns casos, a adulteração é sutil e é apenas detectada com uma combinação de diferentes técnicas, tornando-se um desafio para os métodos oficiais e padrões comerciais. Diversos métodos avançados vêm sendo aplicados em óleos vegetais, para avaliação de fraudes e origem geográfica, incluindo cromatografia líquida de alta eficiência (CLAE), espectroscopia Raman, espectrometria de massas, espectrometria de ressonância magnética nuclear, análise da proporção de isótopo estável de carbono, cromatografia de fluido supercrítico, cromatografia de íons de prata, espectrometria de mobilidade iônica e espectroscopia de infravermelho próximo. Mas a técnica mais empregada na literatura e com bom desempenho é a cromatografia gasosa, através do perfil de ácidos graxos, uma vez que cada óleo vegetal possui um perfil característico [2, 3].

Óleos e gorduras podem facilmente ser contaminados por hidrocarbonetos policíclicos aromáticos (HPAs) devido às características lipofílicas destes compostos. Os HPAs são definidos como compostos orgânicos que possuem dois ou mais anéis aromáticos condensados, formados por carbono e hidrogênio. São produzidos por combustão incompleta da matéria orgânica, por uma série de complexas reações químicas [4]. A principal preocupação relacionada aos HPAs está relacionada à carcinogenicidade. A Agência Internacional de Pesquisas em Câncer (IARC) classifica um dos hidrocarbonetos, o benzo(a)pireno (BaP), no grupo 1, como carcinogênico para humanos, enquanto compostos como benzo(a)antraceno $(\mathrm{BaA})$, benzo(b)fluoranteno $(\mathrm{BbF})$ e criseno (Cri) estão no grupo 2B, possíveis carcinogênicos para humanos [5, 6]. Outros HPAs não cancerígenos podem desempenhar papéis sinérgicos para aumento dos potenciais carcinogênicos e tóxicos de componentes [7].

Considerando a toxicidade relacionada aos HPAs, a presença em alimentos e óleos vegetais vem sendo extensivamente estudada [8]. A contaminação dos alimentos por HPAs pode proceder de diferentes fontes, incluindo rotas ambientais (ar, solo ou água contaminados) ou rotas sintéticas (métodos de cozimento e processamento de alimentos). Os óleos e gorduras podem ser contaminados por poluição atmosférica, durante o processamento tecnológico e pela migração de materiais que entram em contato com o alimento [4]. Os HPAs provenientes de poeira e partículas da poluição atmosférica podem se depositar nas plantas das lavouras e a contaminação ser transferida para o óleo durante a extração. No processamento, a contaminação pode ocorrer devido à secagem da matéria prima, pelo uso de lubrificantes e detergentes, e pela extração dos óleos com solventes. O uso de óleo mineral ou de polietileno reciclado nas embalagens, que apresentem HPAs, podem ser fontes de contaminação [8].

No Brasil, os óleos encapsulados são regulamentados pela Agência Nacional de Vigilância Sanitária (ANVISA). Para óleos, gorduras e creme vegetal, encontra-se em vigor a Resolução $\mathrm{RDC}^{\circ}$ 270/2005, da ANVISA [9], fundamentada nas normas do Codex Alimentarius. Para as normas internacionais de óleos e gorduras, a Codex Stan 210 é aplicada para óleos vegetais específicos, incluindo amostras autênticas de óleos de coco, cártamo, dentre outros, e inclui o perfil de ácidos graxos [10].

Para os HPAs, não existe legislação no Brasil que estipule limites em óleos vegetais, exceto para óleo de bagaço de oliva [11]. Assim, considera-se o Regulamento $n^{\circ}$ 835/2011 da Comunidade Europeia, que preconiza os limites tolerados para os quatro HPAs (BaA, Cri, BbF e $\mathrm{BaP}$ ) utilizados como marcadores da presença destes contaminantes nos alimentos. Para óleos e gorduras vegetais, o limite máximo para o BaP e para a soma dos 4 HPAs é 2,0 e 10,0 $\mu \mathrm{g} / \mathrm{kg}$, respectivamente. Devido à presença proporcionalmente maior de hidrocarbonetos no óleo de coco, a soma permitida para este é de $20,0 \mu \mathrm{g} / \mathrm{kg}$ [12].

O objetivo deste trabalho foi avaliar a identidade e a contaminação por HPAs de óleos encapsulados comercializados na cidade de São Paulo. 


\section{MATERIAL E MÉTODOS}

Foram avaliados 14 óleos apresentados em cápsulas gelatinosas, incluindo óleos de peixe, fígado de bacalhau, prímula (Oenothera biennis L.), girassol (Helianthus annuus L.), linhaça (Linum usitatissimum L.), coco (Cocos nucifera L.), cártamo (Carthamus tinctorius L.), borragem (Borago officinalis L.), chia (Salvia hispanica L.), e misturas dos mesmos: peixe, linhaça e borragem; linhaça, prímula e borragem; cártamo e coco; chia e cártamo; cártamo, coco e chia. A aquisição das amostras ocorreu de forma aleatória, provenientes de diversas localidades da cidade de São Paulo. Após aquisição, as amostras foram conservadas em seus frascos originais, protegidas de luz e estocadas a $4^{\circ} \mathrm{C}$ até às análises.

Foram utilizados padrões individuais de HPAs [benzo(a)antraceno, criseno, benzo(b)fluoranteno e benzo(a)pireno] da marca Supelco e os seguintes padrões de ácidos graxos: mistura com quantidades certificadas de 37 ésteres metílicos de ácidos graxos (EMAG), variando de 4:0 a 24:0, marca Supelco; mistura de EMAG dos isômeros cis-trans do ácido linoléico (18:2) e ácido $\alpha$-linolênico (18:3), marca Sigma; e padrões de EMAG individuais, marca Sigma, incluindo: elaídico (18:1 9t); vacênico (18:1 11c); trans vacênico (18:1 11t); (18:1 7c); (18:1 12c); CLA (18:2 9c,11t e 18:2 10t,12c); palmitoelaídico (16:1 9t), palmítico (16:0); linolelaídico (18:2 9t,12t); EPA (20:5 5c,8c,11c,14c,17c); araquidônico (20:4 5c,8c,11c,14c); docosahexaenóico (20:6 4c, $7 \mathrm{c}, 10 \mathrm{c}, 13 \mathrm{c}, 16 \mathrm{c}, 19 \mathrm{c})$.

Os ácidos graxos foram avaliados como EMAG e quantificados por cromatografia gasosa. Os EMAG foram obtidos usando o método modificado de Hartman e Lago (1973) [13] usando 100 $\mathrm{mg}$ de amostra, $4 \mathrm{~mL}$ de solução de hidróxido de sódio em metanol $(0,5 \mathrm{~mol} / \mathrm{L})$ e $5 \mathrm{~mL}$ de reagente esterificante (solução contendo cloreto de amônio, metanol e ácido sulfúrico) [14]. A quantificação foi realizada em cromatógrafo a gás (CG) com detector de ionização de chama (DIC) e injetor split/splitless modelo GC17A, marca Shimadzu. As condições cromatográficas aplicadas foram: gás de arraste: hidrogênio, pressão constante: $175 \mathrm{kPa}$, volume de injeção: $1 \mu \mathrm{L}$, temperatura do injetor e detector: $250^{\circ} \mathrm{C}$, razão de divisão da amostra: 1:10. A separação dos compostos ocorreu em uma coluna capilar (CP7420, $100 \mathrm{~m}, 0,25 \mathrm{~mm}, 0,25 \mu \mathrm{m}$, Varian) com programação de temperatura: $45^{\circ} \mathrm{C}$ até $175^{\circ} \mathrm{C}\left(12^{\circ} \mathrm{C} / \mathrm{min}\right), 4^{\circ} \mathrm{C} / \mathrm{min}$ até $215^{\circ} \mathrm{C}, 215^{\circ} \mathrm{C}$ por $35 \mathrm{~min}$.

As etapas de análise dos quatro HPAs incluíram extração líquido-líquido, purificação, concentração dos extratos e quantificação, de acordo com a metodologia descrita por da Silva et al. (2017) [15]. Utilizando a extração líquido-líquido, os HPAs foram extraídos do óleo usando n-hexano e solução de N,N-dimetilformamida:água (9:1, v/v), em seguida passou pelo processo de purificação usando cartuchos de extração em fase sólida $(\mathrm{C} 18,500 \mathrm{mg}, 3 \mathrm{~mL}$, Bond Elut, Agilent), com concentração da fase orgânica contendo n-hexano. A quantificação foi realizada por padronização externa em cromatógrafo a líquido de ultra eficiência, marca Shimadzu, com detecção por fluorescência. As condições cromatográficas aplicadas foram: temperatura do forno a $30^{\circ} \mathrm{C}$, comprimentos de onda de excitação e emissão $270 \mathrm{~nm}$ e $390 \mathrm{~nm}$ (para BaA e Cri) e 290 $\mathrm{nm}$ e $430 \mathrm{~nm}$ (para BbF e BaP), coluna cromatográfica Zorbax Eclipse PAH (100 mm x 2,1 mm, $1,8 \mu \mathrm{m}$, Agilent) com coluna de guarda ( $5 \mathrm{~mm} \times 2,1 \mathrm{~mm}, 1,8 \mu \mathrm{m}$, Eclipse Plus, Agilent), fase móvel acetonitrila (A):água (B), com gradiente de eluição: $65 \%$ A por $0,5 \mathrm{~min}$, de 65 a $75 \% \mathrm{~A}$ em $6,1 \mathrm{~min}, 75 \% \mathrm{~A}$ por $10 \mathrm{~min}$, de 75 a $100 \% \mathrm{~A}$ em $3 \mathrm{~min}, 100 \% \mathrm{~A}$ por $4 \mathrm{~min}$ e de 100 a $65 \% \mathrm{~A}$ em 3 min, vazão de $0,4 \mathrm{~mL} / \mathrm{min}$, volume de amostra injetado de $2 \mu \mathrm{L}$, tempo de análise de $27,0 \mathrm{~min}$.

\section{RESULTADOS E DISCUSSÃO}

Quanto ao perfil de ácidos graxos, duas amostras apresentaram perfis de ácidos graxos não característicos, são eles o óleo de coco e o óleo de borragem (Figuras 1 e 2). Sendo que, para o óleo de coco foi observado que os ácidos graxos cáprico, láurico, palmítico, oléico e linoléico estavam com valores diferentes das faixas previstas para estes no Codex Alimentarius [10], enquanto que para o óleo de borragem foram os ácidos graxos linoléico, $\alpha$-linolênico e $\gamma$ linolênico em desacordo com padrões da literatura [16]. Deste modo, não foi possível determinar se houve algum tipo de fraude, pois a alteração das quantidades dos ácidos graxos, em ambos, não revelou ser característica de algum óleo vegetal específico. 


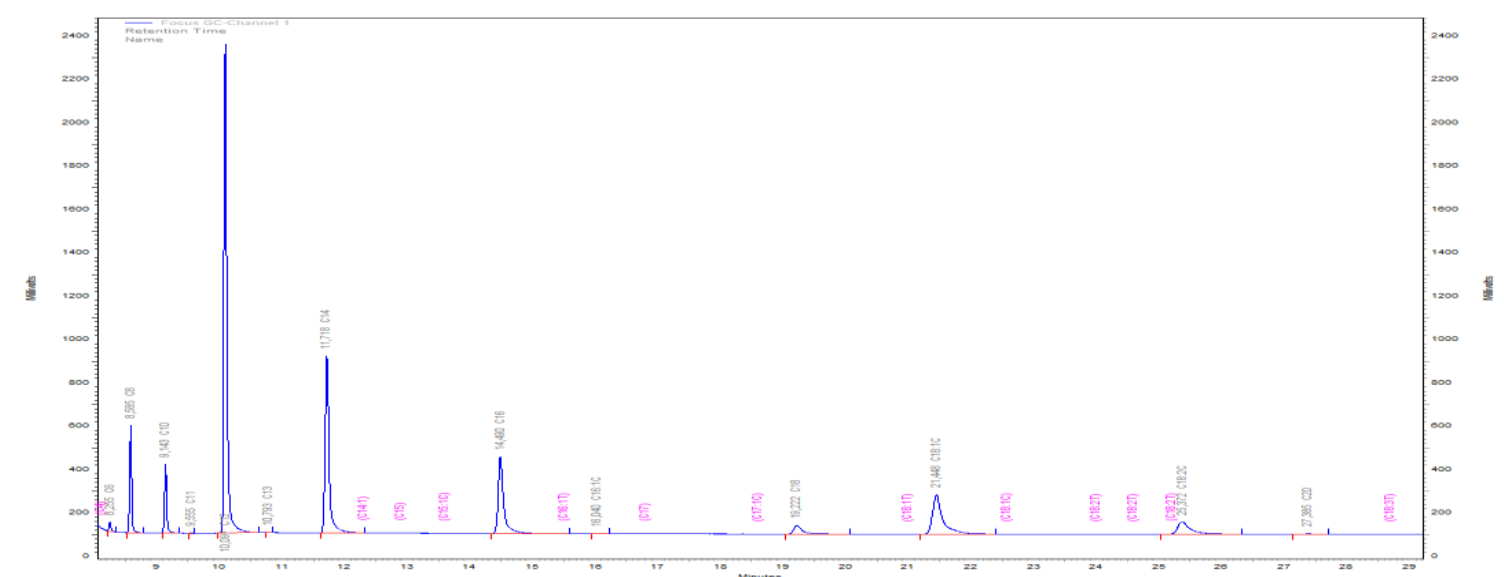

Figura 1: Cromatograma obtido por CG-DIC na análise de ésteres metílicos de ácidos graxos em óleo de coco fraudado.

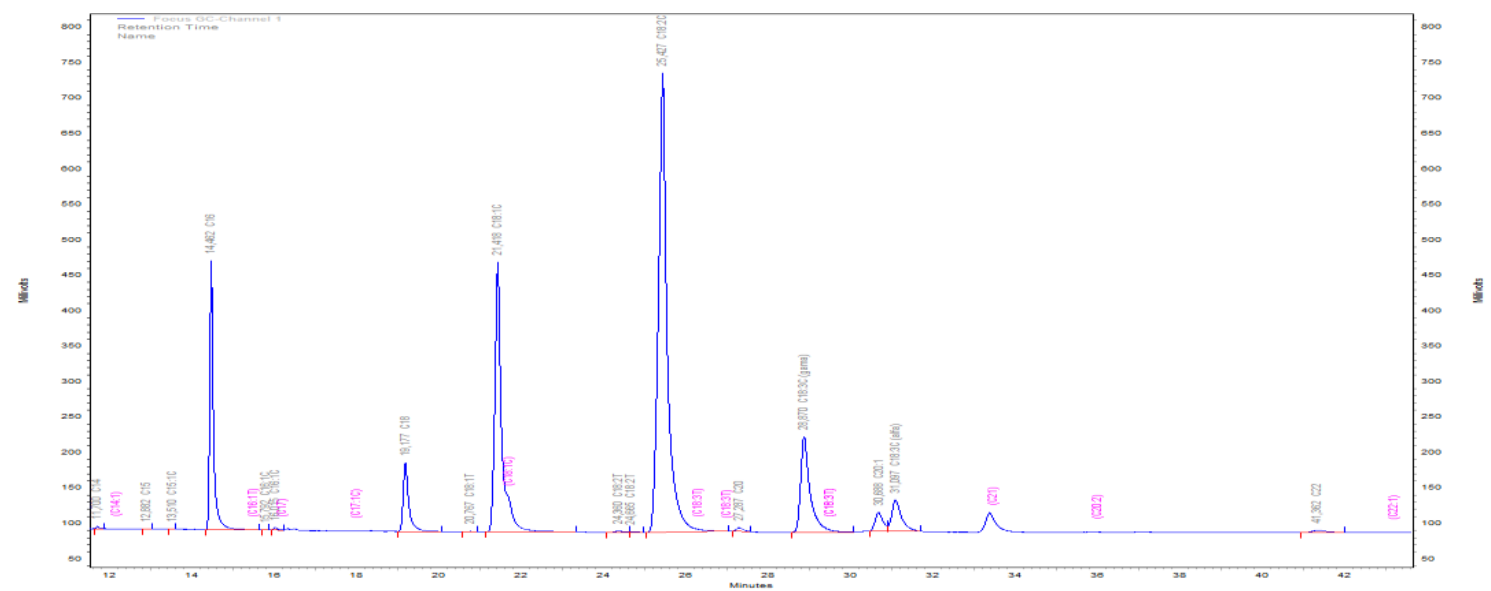

Figura 2: Cromatograma obtido por CG-DIC na análise de ésteres metílicos de ácidos graxos em óleo de borragem fraudado.

Em estudos nacionais já realizados, a avaliação do perfil de ácidos graxos dos óleos demonstrou uma alta incidência de adulteração. Para os óleos encapsulados, 5 amostras de cártamo (5/8), 3 de linhaça (3/6) e 1 de prímula (1/5) estavam adulteradas, e as fraudes ocorreram pela adição de óleo de soja ou pela presença de ácido linoleico conjugado (CLA) [17]. Amostra de óleo de borragem analisada por Leme et al. (2019) [18] apresentou perfil de ácidos graxos semelhante ao encontrado por Hirashima et al. (2013) [17], o que sugere fortemente adulteração pela adição de óleo de soja.

Em trabalho realizado com óleo de coco, uma de cinco amostras tratava-se de uma mistura de óleo de coco e soja [19]. Conforme apresentado por Jamwal et al. (2020) [20], os principais adulterantes do óleo de coco virgem (OCV) são o óleo de mostarda, de parafina (parafina líquida) e de palma. Segundo relatado por Jamwal et al. (2020) [21], a adulteração com parafina é comum em países do sudeste asiático, e a mistura de OCV com 18 a $20 \%$ de parafina não altera notavelmente o sabor ou a cor do produto, mas a mistura pode trazer riscos à saúde humana, uma vez que pode causar distúrbios do intestino, gânglios linfáticos e fígado, e até mesmo câncer.

Os resultados de HPAs encontrados para as amostras comerciais constam na Tabela 1. O hidrocarboneto com maior frequência nas amostras foi o BaA, seguido por Cri. Verifica-se que os níveis foram considerados relativamente baixos e $29 \%$ das amostras (4/14) estavam contaminadas com algum dos HPAs analisados. Pandey et al. (2004) [22] indicaram que o hidrocarboneto mais comumente encontrado nos óleos vegetais analisados na Índia foi o Cri, e 90\% das amostras de óleo de coco e todas de linhaça e cártamo estavam contaminadas com HPAs. 
As concentrações obtidas para o $\mathrm{BaP}$ variaram de abaixo do limite de quantificação (LQ) a $0,51 \mu \mathrm{g} / \mathrm{kg}$ e, para a soma dos $4 \mathrm{HPAs}$, de $<\mathrm{LQ}$ a $5,83 \mu \mathrm{g} / \mathrm{kg}$, estando todas em conformidade com os limites tolerados do Regulamento $\mathrm{n}^{\mathrm{o}} 835 / 2011$ [12]. O óleo de prímula foi o que apresentou maiores concentrações para os quatro HPAs. Comparando-se os resultados deste trabalho com os de outros estudos, os dados foram inferiores aos encontrados por Molle et al. (2017) [23] em óleo de girassol e da Silva et al. (2018) [24] em óleos de cártamo, prímula, linhaça e coco, mas condizentes com os encontrados por Roszko et al. (2012) [25], no qual as concentrações para os quatro HPAs ficaram abaixo de $10 \mu \mathrm{g} / \mathrm{kg}$ para óleos de linhaça e borragem.

Tabela 1: Concentração $(\mu \mathrm{g} / \mathrm{kg})$ de HPAs em óleos encapsulados.

\begin{tabular}{lccccc}
\hline $\mathbf{N}$ & BaA & Cri & BbF & BaP & 4 HPAs \\
\hline $\mathbf{1}$ & $<\mathrm{LQ}$ & $<\mathrm{LQ}$ & $<\mathrm{LQ}$ & $<\mathrm{LQ}$ & $<\mathrm{LQ}$ \\
$\mathbf{2}$ & 0,45 & 0,98 & $<\mathrm{LQ}$ & $<\mathrm{LQ}$ & 1,43 \\
$\mathbf{3}$ & 1,83 & 4,00 & $<\mathrm{LQ}$ & $<\mathrm{LQ}$ & 5,83 \\
$\mathbf{4}$ & $<\mathrm{LQ}$ & $<\mathrm{LQ}$ & $<\mathrm{LQ}$ & $<\mathrm{LQ}$ & $<\mathrm{LQ}$ \\
$\mathbf{5}$ & 1,01 & 0,82 & $<\mathrm{LQ}$ & 0,35 & 2,18 \\
$\mathbf{6}$ & 0,53 & 0,31 & $<\mathrm{LQ}$ & $<\mathrm{LQ}$ & 0,84 \\
$\mathbf{7}$ & 0,42 & $<\mathrm{LQ}$ & $<\mathrm{LQ}$ & $<\mathrm{LQ}$ & 0,42 \\
$\mathbf{8}$ & 0,36 & $<\mathrm{LQ}$ & $<\mathrm{LQ}$ & $<\mathrm{LQ}$ & 0,36 \\
$\mathbf{9}$ & $<\mathrm{LQ}$ & $<\mathrm{LQ}$ & $<\mathrm{LQ}$ & $<\mathrm{LQ}$ & $<\mathrm{LQ}$ \\
$\mathbf{1 0}$ & 1,65 & 0,45 & $<\mathrm{LQ}$ & $<\mathrm{LQ}$ & 2,10 \\
$\mathbf{1 1}$ & 0,27 & 0,43 & $<\mathrm{LQ}$ & $<\mathrm{LQ}$ & 0,70 \\
$\mathbf{1 2}$ & 0,63 & 1,15 & 1,80 & 0,51 & 4,09 \\
$\mathbf{1 3}$ & $<\mathrm{LQ}$ & $<\mathrm{LQ}$ & $<\mathrm{LQ}$ & $<\mathrm{LQ}$ & $<\mathrm{LQ}$ \\
$\mathbf{1 4}$ & $<\mathrm{LQ}$ & $<\mathrm{LQ}$ & 2,94 & $<\mathrm{LQ}$ & $<\mathrm{LQ}$ \\
\hline
\end{tabular}

(BaA: benzo(a)antraceno; Cri: criseno; BbF: benzo(b)fluoranteno; BaP: benzo(a)pireno; HPAs: hidrocarbonetos policíclicos aromáticos; LQ: limite de quantificação: 0,25 $\mu \mathrm{g} / \mathrm{kg}$ para BaA e BaP, 0,30 $\mu \mathrm{g} / \mathrm{kg}$ para Cri e 1,00 $\mu \mathrm{g} / \mathrm{kg}$ para BbF. Amostras: 1 - coco, 2 - cártamo, 3 - prímula, 4 - peixe, 5 - borragem, 6 - chia, 7 - linhaça, 8 - fígado de bacalhau, 9 - peixe, linhaça e borragem, 10 - linhaça, prímula e borragem, 11 - cártamo e coco, 12 - chia e cártamo, 13 - girassol, 14 - cártamo, coco e chia).

Existem diversas justificativas para indicar a presença de HPAs nos óleos, incluindo a contaminação ambiental, seja dos rios e mares, considerando os óleos de peixe, ou do ar, água e solo no qual as plantas foram cultivadas, para óleos vegetais. A poluição do ar contém poeira e partículas com elevada quantidade de HPAs, que podem se depositar na superfície das plantas e contaminá-las, e de forma subsequente os óleos. Este fator torna-se mais significante se a planta é cultivada próxima de áreas industriais e rodovias $[4,26]$.

Outra justificativa para óleos vegetais refere-se ao processamento utilizado para a secagem das sementes. As sementes podem ser secas com ar proveniente de combustão de gases e os HPAs presentes na fumaça podem contaminar as sementes, e de forma consecutiva os óleos, considerando as características lipofílicas destes contaminantes [24, 26].

Mais recentemente, têm-se avaliado que o polietileno reciclado contaminado, presente em garrafas e embalagens plásticas, pode ser uma importante fonte de contaminação, e quanto maior o tempo de armazenamento maiores são as concentrações encontradas. Também o armazenamento da matéria prima em sacos de juta tratados com óleos minerais pode causar a migração de HPAs para os alimentos [8].

\section{CONCLUSÃO}

Os resultados indicaram que tanto a incidência de HPAs quanto a adulteração dos óleos avaliados foram baixos. O monitoramento destes óleos encapsulados deve ser constante, considerando o frequente consumo pela população e a possibilidade dos mesmos terem algum 
tipo de fraude. Boas práticas de fabricação e controle de qualidade devem ser adotados pelas indústrias em todas as etapas de processamento dos óleos, de forma a minimizar o processo de oxidação e contaminação dos óleos.

\section{REFERÊNCIAS BIBLIOGRÁFICAS}

1. Shahid MZ, Imran M, Khan MK, Ahmad MH, Nadeem M, Muhammad N, et al. OMEGA-3 Fatty acids retention, oxidative quality, and sensoric acceptability of spray-dried flaxseed oil. J Food Qual. 2020;2020:72860342020. doi: 10.1155/2020/7286034

2. Salah WA, Nofal M. Review of some adulteration detection techniques of edible oils. J Sci Food Agric. 2021;101(3):811-19. doi: 10.1002/jsfa.10750

3. Xing C, Yuan X, Wu X, Shao X, Yuan J, Yan W. Chemometric classification and quantification of sesame oil adulterated with other vegetable oils based on fatty acids composition by gas chromatography. LWT. 2019;108:437-45. doi: 10.1016/j.lwt.2019.03.085

4. Bansal V, Kim KH. Review of PAH contamination in food products and their health hazards. Environ Int. 2015;84:26-38. doi: 10.1016/j.envint.2015.06.016

5. International Agency for Research on Cancer (IARC), organizer. Some Non-heterocyclic Polycyclic aromatic hydrocarbons and some related exposures. Lyon (FR): IARC; 2010. (IARC Monographs on the evaluation of the carcinogenic risk to humans; vol. 92).

6. International Agency for Research on Cancer (IARC), organizer. A review of human carcinogens. Part F, Chemical agents and related occupations. Lyon (FR): IARC; 2012. (IARC Monographs on the evaluation of the carcinogenic risk to humans; vol. 100F).

7. Iwegbue CM, Ogbuta AA, Otutu JO, Obi G, Egobueze FE, Martincigh BS. Evaluation of human exposure to polycyclic aromatic hydrocarbons from some edible oils and shea butter in Nigeria. Polycycl Aromat Compd. 2021;41(1):109-23. doi: 10.1080/10406638.2019.1570951

8. Bertoz V, Purcaro G, Conchione C, Moret S. A review on the occurrence and analytical determination of PAHs in olive oils. Foods. 2021;10(2):324. doi: 10.3390/foods10020324

9. Brasil. Agência Nacional de Vigilância Sanitária (ANVISA). Resolução RDC nº 270, de 22 de setembro de 2005. Aprova o Regulamento Técnico para óleos vegetais, gorduras vegetais e creme vegetal. Diário Oficial da União. 23 set 2005;184(Seção 1):372-373. Disponível em: https://bvsms.saude.gov.br/bvs/saudelegis/anvisa/2005/rdc0270_22_09_2005.html

10. Codex Alimentarius. Codex Stan CXS 210: Standard for named vegetable oils. Roma (Itália): FAO/WHO; 2019.

11. Brasil. Ministério da Saúde, Agência Nacional de Vigilância Sanitária (ANVISA). Instrução Normativa - IN no 88, de 26 de março de 2021. Estabelece os limites máximos tolerados (LMT) de contaminantes em alimentos. Diário Oficial da União. 31 mar 2021;61(Seção 1):226. Disponível em: https://www.in.gov.br/web/dou/-/instrucao-normativa-in-n-88-de-26-de-marco-de-2021-311655598

12. Commission of the European Communities. Commission Regulation (EU) No. 835/2011 of 19 August 2011 amending Regulation (EC) No 1881/2006 as regards maximum levels for polycyclic aromatic hydrocarbons in foodstuffs. Official J Eur Union. 2011;L215:4-8. Disponível em: https://eurlex.europa.eu/LexUriServ/LexUriServ.do?uri=OJ:L:2011:215:0004:0008:EN:PDF

13. Hartman L, Lago RC. Rapid preparation of fatty acid methyl esters from lipids. Lab Pract. 1973;22(6):475-76

14. Instituto Adolfo Lutz (São Paulo). Métodos físico-químicos para análise de alimentos [Internet]. 4. ed.; 1. ed. digital. São Paulo (SP): Instituto Adolfo Lutz; 2008 [citado em 16 jul 2021]. Disponível em: http://www.ial.sp.gov.br/resources/editorinplace/ial/2016_3_19/analisedealimentosial_2008.pdf

15. da Silva SA, Sampaio GR, Torres EAFS. Optimization and validation of a method using UHPLCfluorescence for the analysis of polycyclic aromatic hydrocarbons in cold-pressed vegetable oils. Food Chem. 2017;221:809-14. doi: 10.1016/j.foodchem.2016.11.098.

16. Firestone D, editor. Physical and chemical characteristics of oils, fats and waxes. Champaign (IL): Aocs Press; 1999.

17. Hirashima K, da Silva SA, Caruso MSF, Aued-Pimentel S. Encapsulated specialty oils commercialized in São Paulo state, Brazil: evaluation of identity (fatty acid profile) and compliance of fatty acids and vitamin E contents with nutrition labeling. Food Sci Tech. 2013;33(1):107-15. doi:10.1590/S010120612013005000022

18. Leme LM, Nakamura F, Tanamati AAC, Valderrama P, Março PH. Fast non-invasive screening to detect fraud in oil capsules. LWT. 2019;109:179-85. doi: 10.1016/j.lwt.2019.03.088 
19. Aued-Pimentel S, Castro FD, de Sousa RJ, Mello MRPA, Abe-Matsumoto LT. Commercialised coconut oil in São Paulo city, Brazil: evaluation of authenticity and nutritional labelling. J Agri Life Sci. 2015;2(1):76-83.

20. Jamwal R, Kumari S, Dhaulaniya AS, Balan B, Kelly S, Cannavan A, et al. Utilizing ATR-FTIR spectroscopy combined with multivariate chemometric modelling for the swift detection of mustard oil adulteration in virgin coconut oil. Vibr Spectrosc. 2020;109:103066. doi: 10.1016/j.vibspec.2020.103066

21. Jamwal R, Kumari S, Dhaulaniya AS, Balan B, Singh DK. Application of ATR-FTIR spectroscopy along with regression modelling for the detection of adulteration of virgin coconut oil with paraffin oil. LWT. 2020;118:108754. doi: 10.1016/j.lwt.2019.108754

22. Pandey MK, Mishra KK, Khanna SK, Das M. Detection of polycyclic aromatic hydrocarbons in commonly consumed edible oils and their likely intake in the Indian population. J Am Oil Chem Soc. 2004;81(12):1131-6. doi: 10.1007/s11746-004-1030-4

23. Molle DR, Abballe C, Gomes FM, Furlani RP, Tfouni SA. Polycyclic aromatic hydrocarbons in canola, sunflower and corn oils and estimated daily intake. Food Control. 2017;81:96-100. doi: 10.1016/j.foodcont.2017.05.045

24. da Silva SA, Torres EAS, de Almeida AP, Sampaio GR. Polycyclic aromatic hydrocarbons content and fatty acids profile in coconut, safflower, evening primrose and linseed oils. Food Chem. 2018;245:798805. doi: 10.1016/j.foodchem.2017.11.109

25. Roszko M, Szterk A, Szymczyk K, Waszkiewicz-Robak B. PAHs, PCBs, PBDEs and pesticides in coldpressed vegetable oils. J Am Oil Chem Soc. 2012;89:389-400. doi: 10.1007/s11746-011-1926-5

26. Sampaio GR, Guizellini GM, da Silva SA, de Almeida AP, Pinaffi-Langley ACC, Rogero MM, et al. Polycyclic aromatic hydrocarbons in foods: biological effects, legislation, occurrence, analytical methods, and strategies to reduce their formation. Int J Mol Sci. 2021;22(11):6010. doi: 10.3390/ijms22116010 